\title{
Exploration-dependent modulation of evoked responses in fascia dentata: Fundamental observations and time course
}

\author{
PATRICIA E. SHARP \\ State University of New York Health Sciences Center at Brooklyn, Brooklyn, New York
}

and

BRUCE L. MCNAUGHTON and CAROL A. BARNES

University of Colorado, Boulder, Colorado

\begin{abstract}
The hippocampal formation is thought to be involved in spatial learning. A role as a more general, intermediate-term memory store has also been suggested. In this report, we address a moderately persistent change in propagation of excitation through hippocampal circuitry which resulted from exposure of animals to environments in which they were free to move and explore. Rats were prepared for chronic recording of perforant path-evoked dentate granule-cell population responses. A large increase in the synaptic component of this response developed over the first several minutes after the animals were transferred to a different but not necessarily novel environment, and decayed with a time constant of about $5 \mathrm{~min}$. This apparent growth in synaptic strength was remarkably well correlated with the animal's recent history of exploratory behavior, and was not due simply to handling or to the electrical stimulation. The amplitude of the population-spike component also varied over time, but was not obviously correlated with changes in the synaptic component. These response changes were different in both apparent mechanism and time course from previously reported, longer lasting, environmentally induced changes in the population-spike component. They also differed from the behavioral state-dependent gating effect reported by Winson and Abzug (1977) to the extent that the present effects long outlasted the behaviors that produced them. Although further analysis is required, it is possible that this phenomenon may reflect one mode of information storage in the hippocampal formation.
\end{abstract}

One of the principal functions of the hippocampal formation appears to be an involvement in the learning of spatial information. Animals with hippocampal damage exhibit a learning impairment in tasks in which the spatial location of a goal is the relevant stimulus dimension (Jarrard, 1983; Morris, Garrud, Rawlins, \& O'Keefe, 1982; Morris, Hagan, \& Rawlins, 1986; O'Keefe \& Nadel, 1978; Olton, Walker, \& Gage, 1978; Sutherland, Wishaw, \& Kolb, 1983). In addition, individual hippocampal pyramidal cells show location-specific firing when an animal navigates through an environment, and thus apparently code information about the animal's current spatial location (O'Keefe, 1976; O'Keefe \& Dostrovsky, 1971). Another role that has been suggested for the hippocampus is that of a temporary (working or intermediateterm) memory store for information of many types (Marr, 1971; Olton, Becker, \& Handlemann, 1980; Rawlins, 1985). These two views of hippocampal function are not

This work was carried out in partial fulfillment of the degree of Doctor of Philosophy by P.E.S. and was supported by NSF Grant BNS8617464 to B.L.M. and PHS Grant AG-03376 to C.A.B. We thank E. J. Green and J. R. Keith for their helpful comments during manuscript preparation. Address correspondence to Bruce L. McNaughton, Department of Psychology, University of Colorado, Boulder, CO 80309. necessarily incompatible. As has been suggested (Rawlins, 1985), "spatial" learning may require temporary storage of information about a wide variety of stimuli as an animal navigates through an environment. If an animal is to form a behaviorally useful representation of an environment as a whole, it seems likely that this would require some type of temporal integration of the sensory consequences of various trajectories through space.

Some insight into how the hippocampal formation may accomplish its postulated roles in learning has come from the study of electrically induced synaptic plasticity in this system. High-frequency, synchronous stimulation of hippocampal afferents results in a long-lasting increase in the amplitude of both the excitatory postsynaptic potential (EPSP) and the spike components of hippocampal population responses (Bliss \& Gardner-Medwin, 1973; Bliss \& Lomo, 1973). This stimulation-induced, long-term enhancement (LTE) can last as long as several weeks (Barnes \& McNaughton, 1980), and its formal properties have made it a promising candidate mechanism for learning and memory storage (McNaughton, 1983; McNaughton, Douglas, \& Goddard, 1978; McNaughton \& Morris, 1987; Swanson, Teyler, \& Thompson, 1982). In addition, studies that have used methods designed to block the development of LTE in the hippocampal formation 
during learning (Morris, Anderson, Lynch, \& Baudry, 1986) or to saturate the LTE-generating mechanism prior to learning (McNaughton, Barnes, Rao, Baldwin, \& Rasmussen, 1986) have shown that these treatments result in deficits on spatial-learning tasks.

If the hippocampal formation uses an LTE-like process to store information during spatial learning, then in principle it might be expected that increases in hippocampal evoked-response amplitude would result from the exposure of freely moving animals to spatially rich environmental input, just as they do from artificial afferent stimulation. One such type of change has been described in earlier reports (Sharp, Barnes, \& McNaughton, 1987; Sharp, McNaughton, \& Barnes, 1985). In those studies, exposure of animals to an enriched environment resulted in a substantial increase in the population-spike component of the evoked response. This increase developed gradually over several days of extensive exposure to the environment and decayed gradually over a period of several weeks after exposure was discontinued. Similar changes in evoked spike amplitude have been reported to result from other behavioral manipulations (Ruthrich, Matthies, \& Ott, 1982; Skelton, Scarth, Wilkie, Miller, \& Philips, 1987).

In this report, we describe an apparently different form of environmental modulation of hippocampal evoked responses, first observed and reported incidentally by Barnes (1979). The effect occurs in conjunction with "exploratory" behavior, which is here operationally defined as a set of behaviors including walking, rearing, turning, and sniffing, and which is associated with the large-amplitude theta-EEG activity in the hippocampal formation (Vanderwolf, 1969).

\section{MATERIALS AND METHOD}

\section{Subjects}

Twenty male, albino, Fischer-344 rats (retired breeders) were obtained from Charles River Laboratories at 9 months of age. Upon arrival, the animals were housed individually in standard colony cages (except where indicated) with lights out between 1700 and $0500 \mathrm{~h}$.

\section{Surgery}

Methods for chronic electrode implantation have been described elsewhere (Barnes, 1979; Sharp et al., 1987). Briefly, Nembutalanesthetized animals were bilaterally implanted with a recording electrode in the hilus of the fascia dentata and a stimulating electrode in the angular bundle (Figure 1A). This enabled recording of the dentate granule-cell population response to single-pulse stimulation of its perforant-path afferents. All electrodes were attached firmly to the skull with dental acrylic and were connected to a small socket which enabled later coupling of the animal to the stimulation and recording equipment.

\section{Histology}

After completion of experimental recording, all animals were perfused transcardially with a $10 \%$ formal saline solution. The brains were sectioned at $40 \mu \mathrm{m}$ to verify electrode placement. All recording electrodes were found to be in or near the hilus, and all stimulating electrodes were in or near the angular bundle.

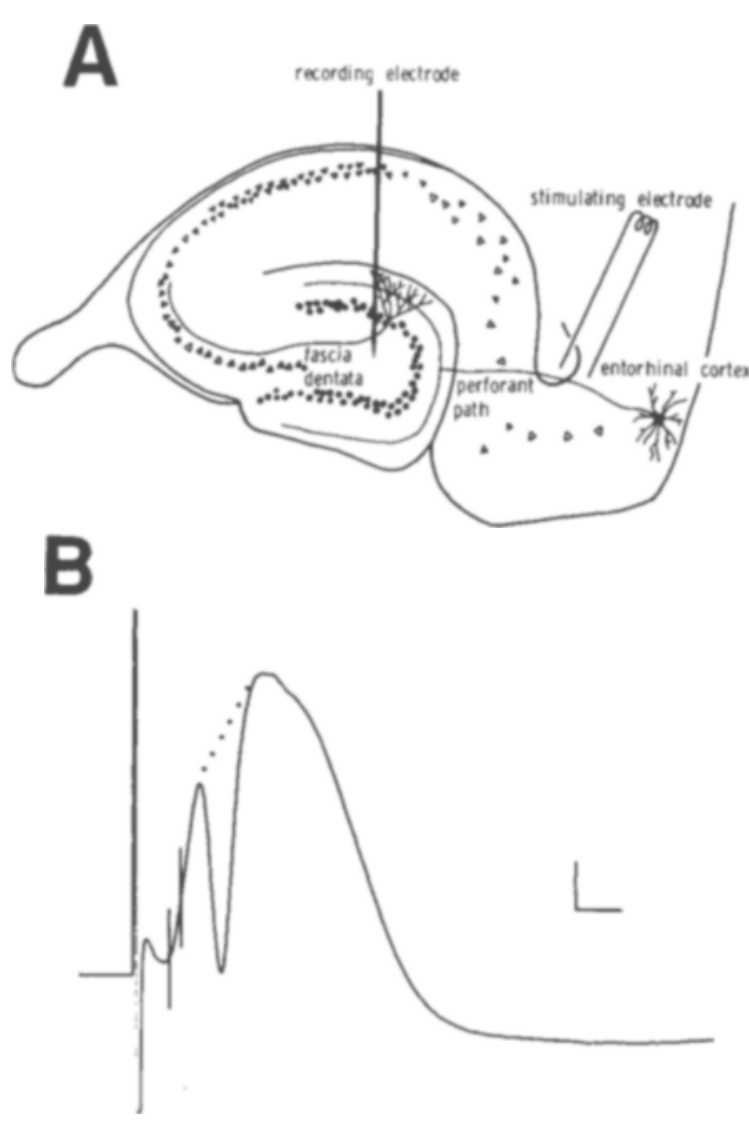

Figure 1. (A) Diagram of the hippocampal formation showing placement of the chronically implanted recording electrode in the hilus of the fascia dentata and the stimulating electrode near the perforant-path fibers in the angular bundle. (B) Example of evoked granule-cell response showing the location of the fixed cursors (vertical bars) for measurement of the synaptic component (field EPSP) of the response and the tangent-line fit between the population-spike onset and offset for the measurement of the population-spike area and latency (dotted line). Calibration is $2 \mathrm{msec}$ and $2 \mathrm{mV}$.

\section{Electrophysiological Stimulation and Recording}

Throughout recording sessions, the connector cap on the animal's head was attached to a $50-\mathrm{cm}$-long stimulation/recording lead. This, in turn, was connected to an easily detachable $5.2-\mathrm{m}$ lead which interfaced with the stimulation and recording equipment. A preamplifier ( $10 \times$ gain) was inserted at the end of the long lead nearest the animal.

Constant voltage, diphasic stimulus pulses $(100 \mu \mathrm{sec}$ per halfcycle) were delivered at a rate of $1 / 10 \mathrm{~Hz}$ to both perforant-path electrodes simultaneously, under the control of a PDP 11/23 computer. Stimulus intensities varied from 0.5 to $10.0 \mathrm{~V}$ under different circumstances. The resulting granule-cell responses were filtered ( $1 / 2$ amplitude at $3 \mathrm{~Hz}$ and $10 \mathrm{kHz}$ ), amplified by a total gain of 100 , and then sampled at $20 \mathrm{kHz}$ per channel by the computer. These response waveforms were stored on magnetic disk for offline analysis.

Figure 1B shows an example of an evoked granule-cell population response. The gradual, positive-going wave is a result of synaptic current flow, whereas the negative wave superimposed on the rising phase of this synaptic response is due to synchronous firing of granule cells. The peak latency of this spike can be interpreted as indicating the approximate median spike latency for individual 
granule cells. The synaptic component (the field EPSP) of the response was measured as the amplitude between two fixed points on the rising phase of the response. The population spike was measured by first fitting a tangent line between onset and offset, and then measuring the area between the line and the curve (see Figure (B). This measure of the integral of the function between spike onset and offset can be taken as a relative indication of the number of granule cells that fire in response to a given stimulus. (Although it can also, in principle, be affected by tonic changes in membrane potential.) This measure was chosen rather than the more commonly used measure of peak amplitude because the former is not influenced as much by the synchrony of cell firing (i.e., the rate of change of the function) as is the latter. It should also be noted that the choice between the two measures is probably relatively unimportant, since both measures have been shown to be highly correlated with each other (Barnes, 1979), and were also seen to parallel each other in this study. The latency to peak of the spike was taken as the point at which the difference between the tangent line and the curve was greatest.

All measures were performed automatically by the computer. To compare responses between animals, the measures for each animal, within each day, were divided by the intercept of a linear regression on the first 10 data points collected on that day (i.e., an estimate of the initial value for that day). Except where noted, response measures are thus expressed relative to a baseline of 1.0.

\section{Behavioral Observations}

During some recording sessions, behavioral observations were taken at the time of each stimulus delivery (i.e., every $10 \mathrm{sec}$ ). Behaviors were divided into three categories: exploratory behaviors, which are defined here as behaviors such as walking, sniffing, and rearing, and which, in rats, are associated with hippocampal thetaEEG activity; inactivity, during which the animal is motionless but not asleep; and grooming. At no time in the recording sessions did the animals exhibit behavioral signs of sleep.

\section{Environments}

During the time that physiological and behavioral recordings were taken, the freely moving, awake animals were always confined to one of three different, relatively small recording chambers or to their home cages.

Chamber 1 was an 18-cm-diameter Plexiglas cylinder, which was placed inside a $63-\mathrm{cm}$-long $\times 61-\mathrm{cm}$-wide $\times 63-\mathrm{cm}$-high shielded wooden frame that was open on one side. Chamber 2 was a $43 \times 22 \times 20 \mathrm{~cm}$ Plexiglas tub inside a $69 \times 41 \times 60 \mathrm{~cm}$ shielded wooden frame and was located in a large room adjacent to that which housed Chamber 1. Chamber 3 was an identical Plexiglas tub located on a table inside a partitioned-off area in the room in which Chamber 2 was located. Home cages used for recording were placed on a table or on the floor inside this same partitioned area. These cages were $53 \times 28 \times 21 \mathrm{~cm}$ and were fitted with black wooden tops with a slit down the middle for the stimulation/recording lead. Up to 6 animals were housed in individual cages in this area at any one time.

\section{Experimental Paradigm}

For each experiment, repeated sessions were conducted during which evoked potentials were recorded at $1 / 10 \mathrm{~Hz}$, as described above, while the animal spent time in one or more of the experimental chambers described above. The order of presentation and the amount of time spent in each environment for each session type of each experiment is summarized in Table 1 . (It should be noted that the animals in Group 2 were also involved in an additional experiment, not reported here, involving repeated, daily recordings while in the home cage. This experiment took place immediately prior to Experiment lb.) As can be seen, some animals participated in more than one experiment; these experimental sessions were separated by a break period during which no recordings were made, and the animals continued to live in their individual home cages and were handled only minimally.

Some sessions (those of Phase 1 of Experiments la and lb) were divided into successive periods of recording from the home cage (HC), with each period being preceded by a brief $(<10$-sec) episode of handling. Such sessions were used to assess the effects of handling alone on response measures. To start a session of this type, the animal's cage top was removed, the animal was picked up, and the 50-cm lead was quickly attached. The animal was then immediately replaced into the $\mathrm{HC}$, the wooden top was replaced, and the lead was connected to the recording apparatus. Simulation and response sampling were begun within $10 \mathrm{sec}$ of the animal having been placed into the $\mathrm{HC}$ and was continued for $10 \mathrm{~min}$. The second part of Phase 1 was then begun. For this phase, the animal's lead was detached from the recording set-up, and the animal was picked up, held for $5 \mathrm{sec}$ on the experimenter's shoulder, and then returned to the HC and reconnected to the recording apparatus. Sampling was resumed and continued for another $15 \mathrm{~min}$. The third HC recording period for Phase I was exactly like the second, except that recording lasted only $10 \mathrm{~min}$, after which the animal was replaced into the $\mathrm{HC}$.

For other session types (such as Phase 2 of Experiments 1a and lb), the animals spent at least some portion of the session in one or more of the recording chambers (RC) described above. These

Table 1

Summary of Experimental Treatment for Each Group of Rats

\begin{tabular}{|c|c|c|c|c|c|}
\hline Experiment & Group & $n$ Animals & $n$ Hemispheres & $\begin{array}{l}\text { Session Type/ } \\
\text { Time (in } \mathrm{min} \text { ) }\end{array}$ & Figure \\
\hline \multicolumn{6}{|l|}{ 1a } \\
\hline Phase 1 & 1 & 4 & 6 & $\mathrm{HC} / 10, \mathrm{HC} / 15, \mathrm{HC} / 10$ & 2 \\
\hline Phase 2 & 1 & 4 & 6 & $\mathrm{HC} / 10, \mathrm{RC} / 15, \mathrm{HC} / 10$ & 2 \\
\hline \multicolumn{6}{|c|}{ 20-day break } \\
\hline 2 & 1 & 4 & 6 & $\mathrm{HC} / 10, \mathrm{RC} / 0.17$ or $90, \mathrm{HC} / 10$ & 4 \\
\hline \multicolumn{6}{|l|}{ lb } \\
\hline Phase 1 & 2 & 6 & 12 & $\mathrm{HC} / 10, \mathrm{HC} / 15, \mathrm{HC} / 10$ & 3 \\
\hline Phase 2 & 2 & 6 & 12 & $\mathrm{HC} / 10, \mathrm{RC} / 15, \mathrm{HC} / 10$ & 3 \\
\hline \multicolumn{6}{|c|}{ 3-day-break } \\
\hline 3 & 2 & 6 & 12 & $\mathrm{HC} / 10, \mathrm{RCl} / 15, \mathrm{HC} / 2, \mathrm{RC} 1$ or $\mathrm{RC} 2 / 15$ & 6 \\
\hline $4 a$ & 3 & 3 & 6 & $\mathrm{RC} / 80$ & 7 \\
\hline $4 \mathrm{~b}$ & 4 & 5 & 10 & $\mathrm{HC} / 15, \mathrm{HC} / 10, \mathrm{RC} / 15$ & 8 \\
\hline
\end{tabular}

Note - HC $=$ home cage; $\mathbf{R C}=$ recording chamber . 
sessions were exactly as just described, except that during handling episodes, the animal was not simply held on the experimenter's shoulder for $5 \mathrm{sec}$, but was also transported to the appropriate environment for subsequent recording.

The RC used for a particular animal was the same throughout its participation in the study, but was Chamber 1, 2, or 3, for different animals. No significant between-chamber effects were observed, and this point is not considered further in the present report.

\section{RESULTS}

\section{Experiment 1a}

This experiment was designed to provide an initial examination of transient changes in evoked responses which had been noticed incidentally in previous studies to follow abrupt (experimenter-induced) changes in spatial location (Barnes, 1979; Sharp et al., 1987, 1985). One aspect of the previously noted changes was a gradual increase in the population EPSP which developed over the first several minutes after the animals were moved to a different location. It was of interest here to assess whether this EPSP change constituted an increase in values above the $\mathrm{HC}$ baseline or a sudden decrease below baseline which then recovered during the ensuing minutes following the spatial transition. Also of interest was the persistence of any changes after the animal was returned to its home cage. Finally, it was important to assess whether the changes were induced simply by the handling of the animals during transport or whether the new environment itself was necessary to induce the changes.

Experiment la was conducted in two phases, as shown in Table 1. During Phase 1, the three consecutive periods of $\mathrm{HC}$ recording (each preceded by a brief episode of handling) enabled an assessment of the effects of handling alone on response amplitude. In Phase 2, when the animal spent a portion of the session in the RC, any additional effects of the RC environment itself could be assessed in relation to the known Phase $1 \mathrm{HC}$ values for this same period. In addition, the $\mathrm{RC}$ values could be assessed in relation to the immediately preceding $\mathrm{HC}$ values for the same session. Finally, any residual effects of RC exposure could be seen in HC values for the period immediately following RC exposure.

Six rats were prepared for evoked-response recording. Fifteen days after surgery, they were moved to the individual HCs described in the Method section. The animals were handled and weighed daily during the subsequent 16-day period prior to the start of recording, at which time daily weighing was discontinued. Because of scheduling requirements, it was necessary to change the animals' lights-out time to between 1900 and $0700 \mathrm{~h}$, so that all recording could be completed before the lights went out. Recording sessions were conducted between the hours of 1300 and 1900 on every other day for an 8-day period, with the subject-order for recording held constant each day. Behavioral observations were taken at the time of each stimulus delivery. Five days prior to the start of the experiment, the animals' evoked potentials were tested on 2 consecutive days, and a stimulus intensity was chosen for subsequent use for each hemisphere which reliably elicited a small population spike (approximately $1 / 3$ maximal).

Responses from several hemispheres were not suitable for spike analysis and so were omitted from the overall data analysis. This left a total of six hemispheres in 4 animals included in the data for Experiment la.

Group means of responses averaged over days of Phases 1 and 2 are shown in Figure 2 for each of the EPSP, spikeamplitude, and spike-latency measures as a function of time. Also shown are the group means, averaged over
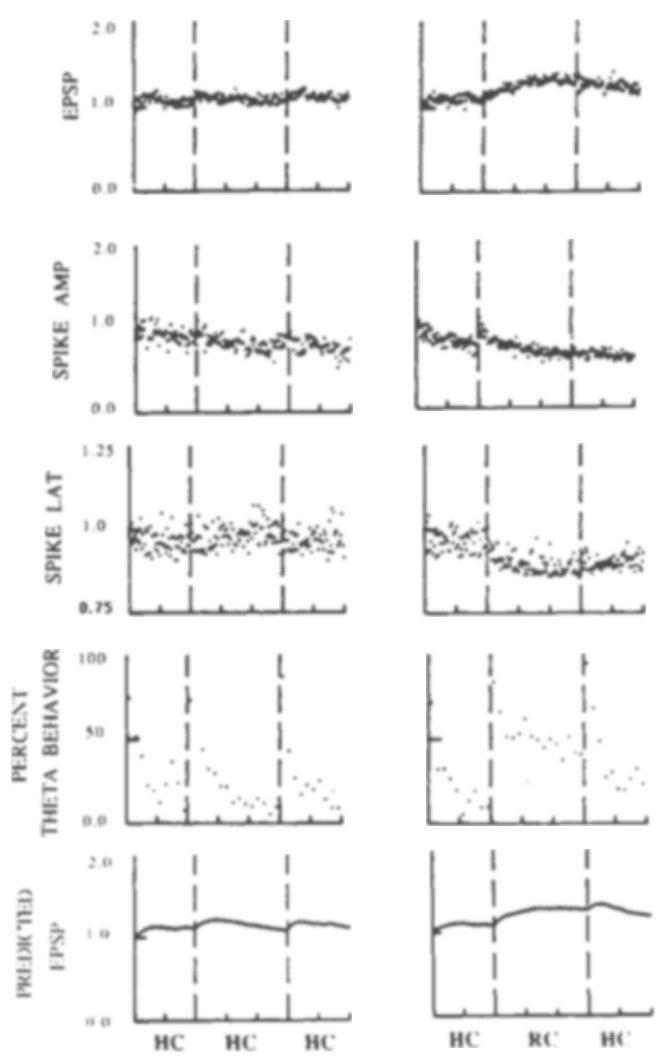

Figure 2. Average normalized values over sessions of dentate-gyrus evoked-potential parameters (EPSP, spike area, and spike latency) in Experiment 1a, plotted as a function of time ( $5 \mathrm{~min} /$ division) for Phase 1 (left) and Phase 2 (right) session types. Responses were normalized by first performing a linear regression on the first $\mathbf{1 0}$ responses of each data type for each hemisphere of each animal in each session. From this, an estimated value at Time 0 was obtained and was used as a divisor for all data points of that type for that hemisphere during that session. The fourth row shows group means for the percentage of observations (taken at each perforant-path stimulus delivery) in each 1-min bin for which the animals showed exploratory behavior. The bottom row shows group means for predicted EPSP values obtained from the application of Equation 1 to individual behavioral records. The values used for $K$ and $\tau$ in Equation 1 were 0.02 and $5 \mathrm{~min}$, respectively. It can be seen that although there is little moment-to-moment correlation between exploratory behavior and response, Equation 1 accounts for a large percentage of the overall variance in the data. This indicates that the behavior over the preceding 5 to $10 \mathrm{~min}$ is a much better predictor of response than is current behavior. The vertical dashed lines indicate the time at which the animals were picked up, handled briefly, and then either replaced into the home cage (HC) or placed into the recording chamber $(\mathbf{R C})$. 
days, for the percentage of observations (grouped into 1-min bins) for which the animals exhibited exploratory behavior. (The nonexploratory behavior exhibited during these sessions fell mostly into the still-alertness category, with grooming being relatively rarely observed.)

During Phase 1, each episode of handling and return to the $\mathrm{HC}$ produced very small, short-lasting increases in EPSP values. Mean values for averages of the last 20 responses in each of the $\mathrm{HC}$ recording segments were $1.04,1.08$, and 1.09 , respectively, thus indicating that the small amount of induced growth had largely disappeared by the end of each of these segments. In contrast, placement into the RC during Phase 2 induced a gradual increase in EPSP above the $\mathrm{HC}$ baseline over the course of $15 \mathrm{~min}$, with a final EPSP growth of 1.29 times baseline. For statistical analysis, an average over days of the means of the last 20 responses of the middle period in each of Phases 1 and 2 was calculated for each hemisphere. An $F$ test (repeated-measures, hemispheres $\times$ phases) showed a significant difference in this measure $[F(1,5)=8.25, p<.05]$. In addition, the increase in EPSP persisted (with some decay) after replacement into the HC, but returned to baseline from day to day.

Surprisingly, changes in the population-spike amplitude were not positively correlated with changes in EPSP. The population spike showed a gradual decline throughout the recording session in both Phase 1 and Phase 2. Superimposed on this overall decline was a transient, abrupt increase at the beginning of the $\mathrm{RC}$ period in Phase 2 and a very slight increase at the beginning of the second $\mathrm{HC}$ period in Phase 1.

Interestingly, although spike amplitude changes did not show the expected relationship to EPSP values, spike $l a$ tency changes did; the latency to peak of the population spike was inversely related to the EPSP values, as would be expected if higher EPSP values were due to larger synaptic currents. During Phase 1, peak latency was relatively stable (although quite variable) throughout the recording session. During Phase 2, introduction to the RC induced a gradual decrease throughout the period, so that the group mean of the last 20 points in the $R C$ was $.88 \pm .06$. After return to the $\mathrm{HC}$ in Phase 2, latency values were still depressed but appeared to recover somewhat over this time.

Each instance of handling induced a brief increase in exploratory behaviors in both Phase 1 and Phase 2. Introduction into the RC during Phase 2 induced a more prolonged increase in this exploratory behavior.

To test whether the repeated RC exposures had any cumulative effects over days, initial HC values for each of the response types were examined over days of Phases 1 and 2. No detectable effects of RC exposures were observed, and these initial values were stable over the course of the experiment.

Relationship between EPSP and behavior. Examination of behavioral state in relation to EPSP amplitude variations over time revealed no obvious moment-to-moment correlation between the two. Thus, at the start of the RC period in Phase 2, there was an immediate, large increase in exploratory behavior which was not accompanied by any such immediate change in EPSP values. In addition, over the remainder of this period, exploration levels decreased, while EPSP values increased, and this is in contrast to the pattern during the subsequent $\mathrm{HC}$ period, in which both EPSP and exploration gradually decreased. However, based on the hypothesis that the EPSP increases could possibly be reflections of information storage, it was reasoned that a somewhat different relationship between the two measures was possible. Specifically, it seemed that the EPSP values may be related not to the animal's momentary behavioral state, but rather, to the animal's recent history of exploration. This seemed likely, since in the static environments used here, the only way for new information to be provided to the system would be as a result of the animal's own exploratory behaviors. In the absence of such behavior, all input to the sensory organs would be almost completely static. Thus, it was reasoned that each epoch of exploratory behavior might result in a small increase in EPSP amplitude, and that such increases would be additive over epochs (with each increase hypothetically reflecting storage of an additional, small set of environmental information). Since EPSP growth was quite short-lasting, it was further assumed that such cumulative increases would decay exponentially (presumably corresponding to rapid "forgetting" of the information). These assumptions can be expressed as

$$
\operatorname{EPSP}(t)=C+K B(t)+G(t-\Delta t) e^{-\Delta t / r},
$$

where $\operatorname{EPSP}(t)$ is the amplitude of EPSP at time $t ; C$ is a baseline level of EPSP strength (the initial EPSP value determined by linear regression as described above); $K$ is a constant found empirically; $\mathrm{B}(t)$ represents behavior at time $t$, and is always either 1 (for exploratory behavior) or 0 (any nonexploratory behavior); $G(t-\Delta t)$ is the proportional growth over baseline of the EPSP present on the last observation; $\Delta t$ is the time since the last observation; and $\tau$ is the empirically determined decay-time constant. This is essentially the numerical description of a parallel RC circuit (i.e., a "leaky" integrator the output of which depends on the history of input over the intervals of the order of $\tau$, rather than only on the current input). To test the model, a predicted set of EPSP values was generated for each data set of each hemisphere on the basis of the animal's behavior for that recording session. The value for $C$ was 1.0 , the normalized initial HC value. The value for $\tau$ was chosen by examining individual data sets in which animals showed very low exploratory activity levels subsequent to a previous EPSP increment. This enabled an estimate of how fast the response decayed in the absence of exploratory behavior. Such examinations revealed that EPSP increments decayed to about one third of their initial value after approximately $5 \mathrm{~min}$. Thus, $5 \mathrm{~min}$ was chosen as the value of $\tau$. The value for $K$ was then found empirically. A value of 0.02 was found to result in the best fit to the data as assessed by eye. The means of the predicted values using these parameters are 
shown in the bottom row of Figure 2. Although clearly not accounting for all of the experimental variance, the values derived from the model using the behavioral data as input predicted the EPSP amplitudes reasonably well, certainly much better than any model based solely on the instantaneous behavioral state.

\section{Experiment 1b}

A replication of Experiment la was carried out using a different set of animals $(n=6$, with two hemispheres per animal included for analysis). The conditions of the experiment were essentially the same.

The results for EPSP and spike were very similar to those of Experiment 1a, except that somewhat more growth in EPSP was observed in the Phase $1 \mathrm{HC}$ conditions. The relationship between exploratory behavior and EPSP growth was assessed using Equation 1 and the same values for the growth constant, $K$, and the decay-time constant, $\tau$, as were used in Experiment 1a. The predicted and observed values, as well as the behavioral observations on which the predicted values are based, are shown in Figure 3. These parameters produced a surprisingly good fit to the overall data pattern.

To illustrate the magnitude and robustness of the EPSP changes for individual animals, the individual data from Experiments $1 \mathrm{a}$ and $1 \mathrm{~b}$ are presented in Table 2. For this, Phase 2 data for each animal were used to calculate an initial $\mathrm{HC}$ value and a terminal $\mathrm{RC}$ value (the average of the last 20 responses in the $\mathrm{RC}$ ) for each animal, aver-

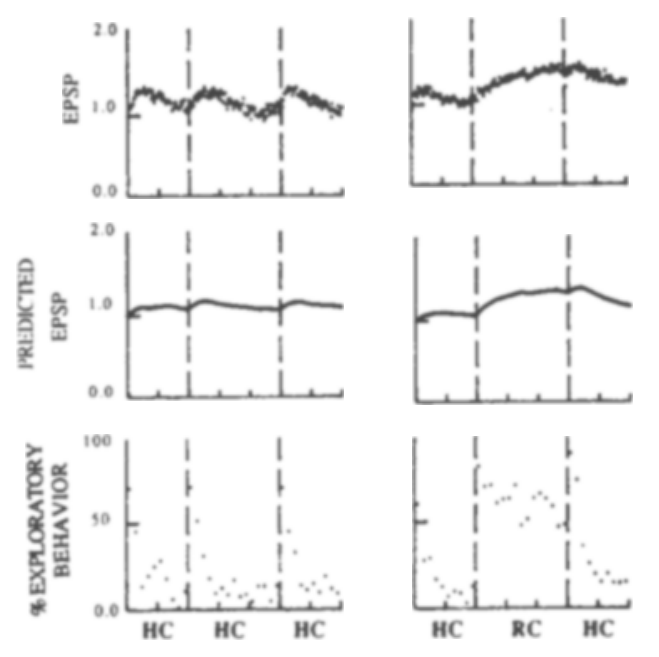

Figure 3. Average normalized values over sessions of dentate-gyrus evoked-potential EPSP values (top row), group means of percentage of observations during which exploratory behavior occurred averaged over 1-min bins (bottom row), and predicted EPSP values obtained from the application of Equation 1 to individual behavioral records (middle row) in Experiment 1b, plotted as a function of time $(5 \mathrm{~min} / \mathrm{small}$ division). Values used in Equation 1 for $K$ and $\tau$ were 0.02 and $5 \mathrm{~min}$, respectively. Use of this temporal-integration model again provided a much better fit to the overall data pattern than could be obtained by taking into account only the instantaneous behavioral state. The vertical dashed lines indicate the time at which the animals were picked up, handled briefly, and then either replaced into the home cage (HC) or placed into the recording chamber (RC).
Table 2

Individual Animal Data for EPSP Amplitude for Phase 2 of Experiments 1a and 1b

\begin{tabular}{cccc}
\hline Animal & Begin HC & End RC & Fractional Change \\
\hline 2 & 0.42 & 0.50 & 1.19 \\
4 & 0.25 & 0.30 & 1.20 \\
5 & 0.73 & 1.10 & 1.51 \\
7 & 1.87 & 2.15 & 1.15 \\
38 & 0.28 & 0.38 & 1.36 \\
39 & 0.42 & 0.49 & 1.17 \\
40 & 0.72 & 1.04 & 1.44 \\
41 & 2.65 & 3.70 & 1.40 \\
42 & 0.89 & 1.48 & 1.66 \\
43 & 1.55 & 1.99 & 1.28 \\
Mean & 0.98 & 1.31 & 1.34 \\
SD & \pm 0.80 & \pm 1.07 & \pm 0.17 \\
\hline
\end{tabular}

Note-Each row shows absolute values for initial home cage (Begin $\mathrm{HC}$ ) and terminal recording chamber (End RC) time points, as well as the relative growth for these points, averaged over hemispheres and sessions for an individual animal.

aged over hemispheres and sessions. Although the absolute values differ considerably between animals, partly as a result of electrode location and partly due to the position on the rising phase on the EPSP at which the response was measured, the environmentally induced increase is clearly quite reliable. The mean proportional growth over baseline for the two experiments was $1.34 \pm 0.17$.

\section{Experiment 2}

In Experiment 1, exposure to the relatively novel RC induced a gradual growth in EPSP that was greater than that resulting from handling only. Experiment 2 was designed to assess whether this RC effect could be induced by a very brief exposure to the $\mathrm{RC}$, or whether it required the continued presence of the animal in the RC. Also of interest was whether EPSP growth induced in the RC would remain over a prolonged period of continued exposure to the RC.

In this study, the animals from Experiment la received a 20-day break period and then were tested during recording sessions like those used in Phase 2 of that experiment (see Table 1), except that in the present experiment, the length of the RC period was varied, so that it was either $10 \mathrm{sec}$ or $90 \mathrm{~min}$. Alternating sessions of short and long $\mathrm{RC}$ exposure were administered to each animal every other day, with appropriate counterbalancing. Four sessions of each type were administered to each animal. The evoked response for one animal deteriorated during this time, and so could not be used for subsequent analysis.

Because the interpretation represented by Equation 1 was not developed until after this experiment had been completed, the full importance of behavioral measures was not yet appreciated, and these measures were omitted in this experiment.

Group means of normalized values averaged over each of the two types of recording session are shown in Figure 4. The 10-sec RC exposures were too brief to permit any recordings during this time, so data from these sessions (left) show just the two consecutive HC periods that surrounded this exposure. 

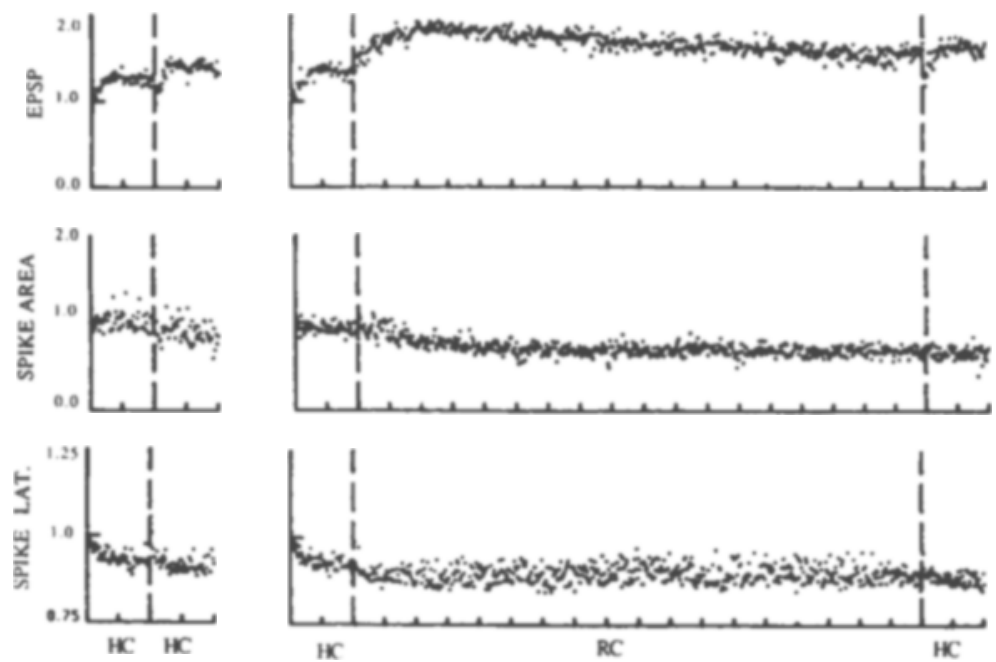

Figure 4. Average normalized values over sessions of dentate-gyrus evoked-potential parameters (EPSP, spike area, and spike latency) in Experiment 2, plotted as a function of time ( $5 \mathrm{~min} /$ division) over each of the two session types (average is over five hemispheres). For the session types involving the short (10-sec) RC exposure time (left), the RC period was too brief to allow any recordings in the RC itself. Thus, this exposure is not indicated even though it did, in fact, intervene between the two HC periods shown. The vertical dashed lines indicate the time at which the animals were picked up, handled briefly, and then either replaced into the home cage (HC) or placed into the recording chamber (RC).

The 90-min RC exposure induced a larger total EPSP growth than did the 10-sec exposure. The average of the last 20 EPSP values during the second $\mathrm{HC}$ period in the $10-\mathrm{sec} \mathrm{RC}$ exposure condition was 1.36 , whereas that for the comparable time period during the 90 -min RC exposure was 2.09 , and this difference was statistically significant $[F(1,4)=26.4, p<.005]$. The fact that the 10 sec exposure did not induce the full EPSP effect provides conclusive evidence that there is at least a part of the EPSP growth that is not induced just by the handling and transport involved in moving the animal to the RC. EPSP growth during the 90-min exposure reached its peak after about $10 \mathrm{~min}$. After this, EPSP values began to decline slowly to a value of 1.56 by the end of the 90 -min RC exposure. The 10-sec RC exposure also appeared to induce a small growth of EPSP. The EPSP value for the last 20 responses in the second $\mathrm{HC}$ period $(1.36 \pm .10)$ was somewhat higher than that for the last 20 responses

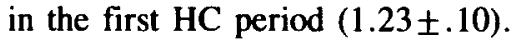

In both types of session, the return to the $\mathrm{HC}$ after $\mathrm{RC}$ exposure resulted in an abrupt decrease in EPSP, relative to the last recorded values. The average decrease was 0.18 . This sudden decline is considered in more detail in Experiment 3.

The results for the recordings during the HC periods in this experiment were different from those for the same animals in Experiment 1a, in that each placement into the $\mathrm{HC}$ resulted in a relatively large growth in EPSP. The average value for the end of the initial HC period (collapsed over conditions) in this experiment was $1.26 \pm .06$, whereas that in Experiment la was 1.06士.03. It is possible that this discrepancy might be explained by the differential amount of handling received by the animals preceding each of the two experiments. Prior to the start of Experiment 1a, the animals had been handled on a daily basis for a long period of time. In contrast, the rats were handled only minimally during the weeks prior to the start of Experiment 2. The lengthy period of handling and replacement into the $\mathrm{HC}$ prior to Experiment la may have caused the animals to habituate to this treatment prior to the start of the recording sessions. The lack of effect of such treatments on EPSP values may have been related to this behavioral habituation. Conversely, the lengthy period of relatively little handling prior to Experiment 2 may have resulted in loss of habituation. This explanation seems particularly plausible given the empirical relationship seen in Experiment 1 between exploratory behavior and EPSP growth. Thus, if animals have become habituated to handling and replacement into the $\mathrm{HC}$, then they might be expected to generate relatively little exploratory behavior subsequently, and thus show little EPSP growth.

As in Experiment 1, the latency to peak values for the spike were approximately inversely related to the EPSP changes.

Spike-area values declined gradually over the 20 -min period for the 10-sec RC sessions and over approximately the first 30 min of the $90-$ min RC sessions. They remained relatively stable for the remainder of the session. The 90 min RC exposure appears to have induced a greater de- 
crease in spike amplitude values than did the 10-sec exposure, although this difference did not reach statistical significance.

\section{Experiment 3}

In the preceding experiments, abrupt decreases in EPSP values were sometimes associated with the return of the animal to the $\mathrm{HC}$ after exposure to the RC. A clear example of this is illustrated in Figure 5, in which the EPSP data from the transitional periods of Experiment 2 are shown on an expanded scale. This sudden decline was not observed on transitions from $\mathrm{HC}$ to RC. Experiment 3 was designed to clarify the enabling conditions for this effect. It may be that a decline occurs whenever an animal returns to a place it has experienced in the recent past or whenever it is moved to a relatively familiar location (such as the HC). Alternatively, it may be that any transfer that occurs when EPSP is already elevated will cause a reduction, regardless of the location to which the animal is transferred.

The animals from Experiment $1 \mathrm{~b}$ were given alternating sessions of two types. Both types involved an initial three periods consisting of $10 \mathrm{~min}$ in the $\mathrm{HC}, 15 \mathrm{~min}$ in the same RC as in Experiment $1 \mathrm{~b}(\mathrm{RC} 1)$, followed by a return to the $\mathrm{HC}$ for $2 \mathrm{~min}$. The animals were then placed either into RC2 (to which they had not yet been exposed that day) or back into RC1. The two session types were given on alternate days over a 4-day period. The chambers used for RC1 and RC2 were counterbalanced across subjects. Behavioral observations were made during the first session of each type.

Mean EPSP and behavioral data are shown in Figure 6. Placement into the $\mathrm{HC}$ after $\mathrm{RC} 1$ exposure induced a slight, abrupt reduction in EPSP values, just as it had in the previous experiments. The mean over both session types for the last 20 EPSP values during the first RC1 period was $1.37 \pm .03$, whereas that for the following 2-min HC period was $1.31 \pm .04$. Subsequent placement into $\mathrm{RC} 2$ and return to $\mathrm{RC} 1$ both induced a further EPSP decrease, with initial $R C 2$ and $R C 1$ values of $1.17 \pm .06$
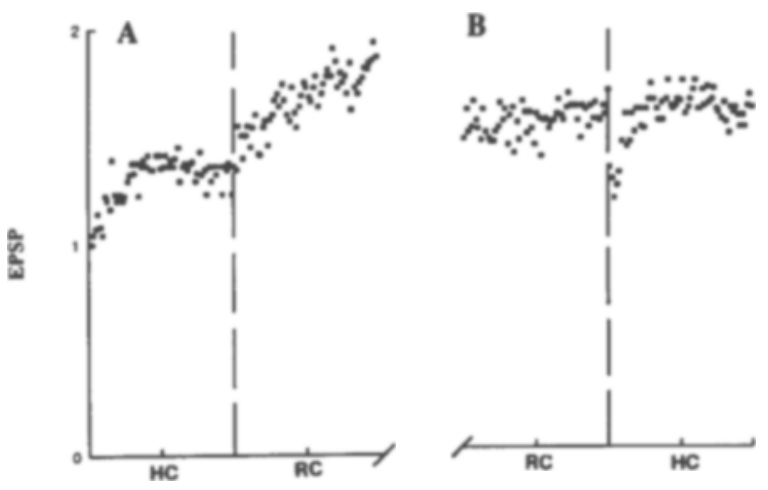

Figure 5. Expanded EPSP data from the transitional periods of Experiment 2 (see Figure 4). (A) Transition from home cage (HC) to recording chamber (RC), with no sudden EPSP drop. (B) Transition from RC to HC, showing the sudden EPSP decrease.
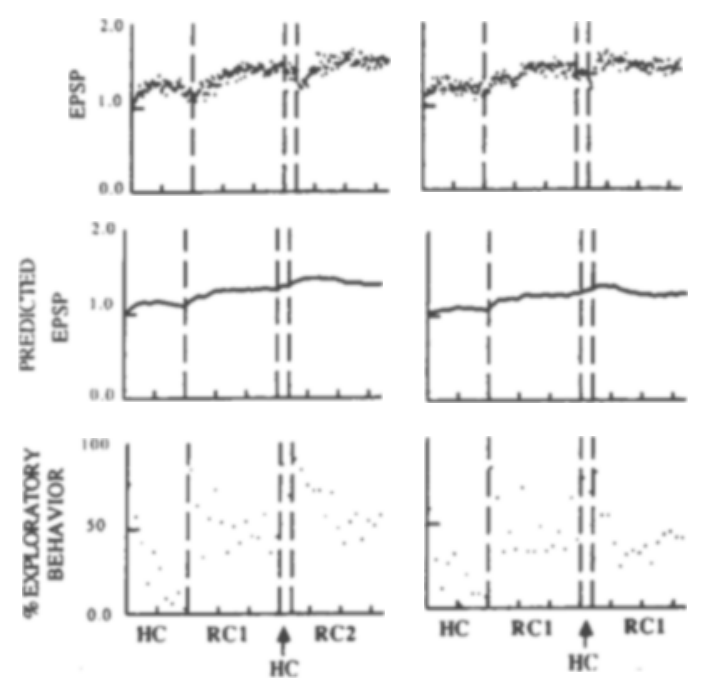

Figure 6. Group means over days of normalized EPSP values (top row) and percentage of observations during which exploratory behavior occurred averaged over 1-min bins (bottom row) in Experiment 3, plotted as a function of time (5 $\mathrm{min} / \mathrm{small}$ division). The middle row shows group means for predicted EPSP values obtained from the application of Equation 1 to individual behavioral records. The values used for $K$ and $\tau$ in Equation 1 were 0.02 and $5 \mathrm{~min}$, respectively. The vertical dashed lines indicate the times at which the animals were picked up, handled briefly, and then either replaced into the home cage (HC) or placed into Recording Chamber 1 or 2 (RC1, RC2). Note that sudden reductions in EPSP amplitude, like those illustrated in Figure 5, occur only when exploratory-dependent growth of EPSP is present.

and $1.20 \pm .04$, respectively. This decrease was somewhat more persistent in the case of RC2. The similarity of the decreases suggests that the phenomenon is not related to the features of the environment to which the animal is transferred. Rather, it appears to depend on the level of EPSP prior to the time that the transfer is made. Further analysis of this phenomenon will be presented in a related paper (Green, McNaughton, \& Barnes, in press).

Application of Equation 1 to the data of this experiment (Figure 6, middle row) using the previous value of the growth constant, $K$, predicted the overall pattern of EPSP changes quite well. Note, however, that the equation does not take into consideration the transient EPSP reduction.

\section{Experiment 4a}

In the experiments reported so far, the onset of electrical test stimulation coincided with the occurrence of the behavioral treatment of handling and attachment of the stimulation/recording lead. Thus, any effects on the evoked potentials that may have resulted from the perforantpath stimulation itself could have been confounded with the effects of the behavioral treatments. Experiment $4 \mathrm{a}$ was designed to examine the effects of stimulation per se, independent of the effects of behavioral manipulation.

Three animals received alternating sessions of two types over a 6-day period. In both conditions, they were connected to the recording equipment and were placed into 
an RC for $80 \mathrm{~min}$. In Condition 1, perforant-path stimulation at $1 / 10 \mathrm{~Hz}$ began immediately. In Condition 2, stimuli were delivered only for the last $20 \mathrm{~min}$.

Group mean data for the two session types are shown in Figure 7. Normalized values for Condition 2 were obtained by dividing by the average start value for the three Condition 1 sessions. The mean values and overall pattern of EPSP, spike area, and spike latencies obtained over the last $20 \mathrm{~min}$ were nearly identical for the two session types [EPSP: $(F(1,5)=.50 ; p>.25$; spike amplitude: $F(1,5)=5.00 ; p>.05 ;$ and spike latency: $F(1,5)=.71$; $p>.25]$. Thus, perforant-path stimulation itself did not significantly affect response amplitude.

\section{Experiment 4b}

As a further test of the possible effects of perforantpath stimulation per se, a group of 5 animals received perforant-path recording sessions prior to any daily handling or other contact with the experimenter. For this experiment, the animals were plugged into the recording and stimulation equipment and left undisturbed in the $\mathrm{HC}$ for at least $15 \mathrm{~h}$. Responses were then recorded in the $\mathrm{HC}$ for 15 min ('PRE-HC.' in Figure 8). This was followed by a brief handling treatment, an additional $10 \mathrm{~min}$ of recording in the $\mathrm{HC}$, and $15 \mathrm{~min}$ of recording in the $\mathrm{RC}$, as in the previous experiments.

The results are shown in Figure 8. Although there were slight, gradual decreases in both the EPSP and spikeamplitude values over the prehandling $\mathrm{HC}$ period, these clearly differed in pattern from changes induced by the subsequent $\mathrm{HC}$ and $\mathrm{RC}$ treatments.

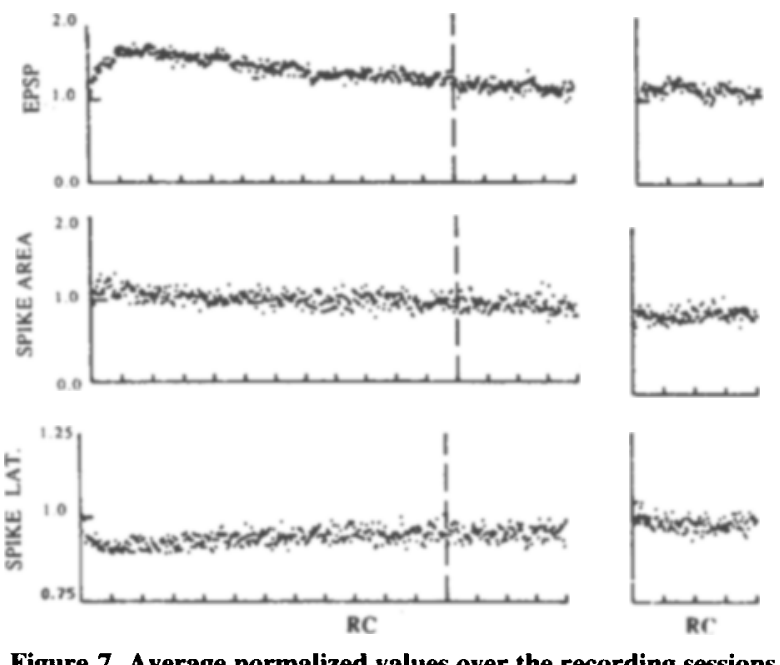

Figure 7. Average normalized values over the recording sessions of dentate-gyrus evoked-potential parameters (EPSP, spike area, and spike latency) in Experiment 4, plotted as a function of time (5 $\mathrm{min} /$ division). Single perforant-path test stimuli were administered either throughout the $\mathbf{8 0}$-min session in the recording chamber (RC, left) or only during the last $\mathbf{2 0}$ min (right). The vertical line in the left column demarcates the beginning of the last $20 \mathrm{~min}$ of the continuous recording session, which began after $60 \mathrm{~min}$ of continuous exposure to the $\mathrm{RC}$, for comparison with the right-hand column. Values for the last $\mathbf{2 0}$ min were not significantly different between the two conditions.

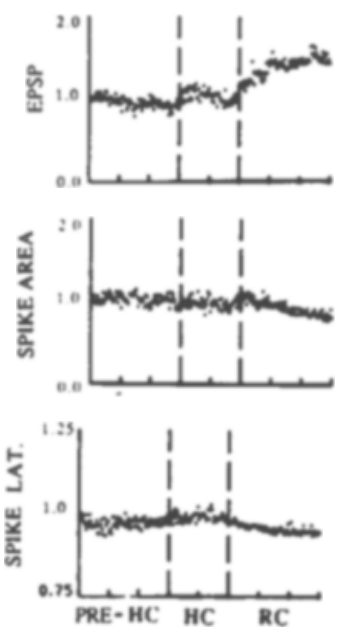

Figure 8. Average normalized values for the dentategyrus evokedpotential parameters (EPSP, spike area, and spike latency) in Experiment $4 \mathrm{~h}$, plotted as a function of time $(5 \mathrm{~min} /$ division). Sessions consisted of an initial prehandling home cage period (pre-HC) followed by subsequent $\mathrm{HC}$ and recording chamber (RC) periods as in the preceding experiments. These results show that the large EPSP effect results from exposure to the relatively novel $\mathrm{RC}$ and are not due to stimulation per se, changes in the sleep-wakefulness state, or "arousal" associated with handling.

\section{DISCUSSION}

The experiments reported here demonstrate a relatively short-lasting form of plasticity in the perforant pathevoked dentate granule-cell response that was correlated with the animals' recent histories of exploratory behavior in an environment. The population EPSP component of this response showed a substantial, cumulative increase above baseline during bouts of exploratory behavior, whether such behavior took place in the home cage or in a relatively novel environment, and these increases lasted for a period of several minutes after cessation of the exploratory behavior itself. It should be noted that this temporal pattern of behaviorally related changes is different from the moment-to-moment correlations between behavior and these responses that have been reported earlier (Winson \& Abzug, 1977, 1978) (see discussion below). The largest EPSP increases (as well as the most persistent bouts of exploratory behavior) resulted after the transfer of animals from one environment to another, although handling alone was also followed by modest increases (Experiments $1 \mathrm{a}$ and $\mathrm{lb}$ ). Experiment 2 demonstrated conclusively that the full effect of transfer to a new environment was not due just to the handling and transport involved in this manipulation, but required continued presence in the relatively new environment. This was shown by the fact that when the animals were transported to a new environment and then back to their home cages within a brief period, subsequent EPSP growth was not as large as when they were transported only once, to the new environment, and allowed to remain there. Experiments $4 a$ and $4 b$ demonstrated that the change was not due to the perforant-path test stimulation itself. 
It should be noted that this change was generally larger than those, such as LTE (see introduction), which result from high-frequency electrical stimulation, and was at least as reliable.

A reduction in the latency to peak of the population spike also resulted from the environmental manipulations. This latency change was generally inversely related to the EPSP change, suggesting that there is a population of cells that fire at shorter latency in response to perforant-path stimulation, as would be expected from the EPSP growth.

Surprisingly, the observed changes in the size of the population spike did not parallel the EPSP or spike-latency changes in any simple manner. Spike values generally declined over the first 20 to 30 min of all recording sessions involving manipulation of the animal. Thus, even though EPSP values were generally increasing over this time, the number of granule cells firing as a result of this synaptic activation showed a simultaneous decrease. Superimposed transient spike increases sometimes accompanied transfers from one environment to another, usually when the transfer was to a relatively novel environment. The magnitude of the spike decline tended to be larger in a relatively novel environment.

The observed empirical relationship between exploratory behavior and EPSP increases was demonstrated by applying Equation 1, which assumes that each epoch of exploratory behavior is associated with a fixed increase in EPSP amplitude and that the cumulative increase decays with an exponential time constant of about $5 \mathrm{~min}$. Application of this equation to the behavioral data provided surprisingly good predictions of the EPSP changes across a variety of experimental manipulations. There are several possible general explanations for why this particular empirical relationship should exist. One is that the growth may be strictly related to motor activity or to hippocampal theta-EEG activity (known to accompany exploratory behavior) per se. This possibility seems less likely in light of data presented in a related paper (Green et al., in press) showing that when motor behavior (and the accompanying hippocampal theta-EEG activity) is induced by turning on a treadmill under an animal's feet, the relationship seen here between motor behavior and EPSP increases is not observed. Another possibility is that the exploratory activity observed here is just one index of a nonspecific systemic change, possibly in hormone levels or in the reticular activating system, such as might be termed "arousal," and that these changes are also responsible for the EPSP increases. This possibility cannot be ruled out by the present data, although results reported by Green et al. have suggested that a variety of treatments that might be considered on an a priori basis to result in considerable levels of arousal do not necessarily, in themselves, result in substantial EPSP increases. Another possible interpretation, and the one favored here, is that the EPSP growth is somehow related to the continuing input of environmental sensory information that accompanies exploration. According to this view, each increment reflects the storage of the small amount of new information made available by the shift in the animal's sensorium with each brief epoch of exploratory behavior. Each such set of new information is then lost (or "forgotten") over the next few minutes. This explanation is compatible with the fact that forcing animals to run on a treadmill in the Green et al. study was not accompanied by substantial EPSP growth. Even though treadmill walking both induced theta activity and was probably quite arousing, it did not provide input of new spatial information. It should also be noted that this interpretation is consistent with the fact that exploration-related increases are seen in the HC, even though this environment is not at all novel. The rapid decay of information from this system means that even HC-related information would, in fact, be "new" to this system, provided that the animal had been relatively quiescent prior to the experimental manipulations.

In some cases, an abrupt and transient decline in EPSP values accompanied the transfer of animals from one environment to another. In Experiment 3, it was shown that this phenomenon occurred only in the presence of prior exploration-related EPSP growth. It is not obvious why this should be the case, but it suggests that processes occurring immediately after a transition interact somehow with processes related to exploration-induced growth to cause the drop.

Possible mechanisms. There are at least two general categories of mechanism that might account for the observed growth of the extracellularly recorded EPSP: (1) increased synaptic conductance and (2) increased synaptic driving potential, such as what might accompany a pronounced hyperpolarization of the granule cells. Apparently contrary to either such simple interpretation, however, is the fact that the decline in latency of the population spike is consistent with the first class of mechanism, but not with the second, whereas the decline in spike amplitude is consistent with the second class of mechanism, but not with the first. As a general explanation for this overall pattern of results, it should be noted that numerous factors, such as momentary behavioral state, and activity levels in subcortical areas projecting to the hippocampus have been shown to affect the population spike for these responses, and often do so in the absence of any effects on EPSP values (Abraham \& Goddard, 1982; Alvarez-Leefmans \& Gardner-Medwin, 1975; Assaf, Mason, \& Miller, 1979; Assaf \& Miller, 1978; Dahl \& Winson, 1986; Fantie \& Goddard, 1982; Green, Barnes, \& McNaughton, 1986; Robinson \& Racine, 1986; Winson, 1980; Winson \& Abzug, 1977, 1978; Winson \& Dahl, 1985). Changes in many of these factors almost certainly occur as a result of the behavioral manipulations used here. Since these factors generally do not affect EPSP values, they could cause changes orthogonal to those seen here for the EPSPs, and could possibly mask the predicted positive correlation between EPSP and spike values. In addition, dentate inhibitory interneurons are known to show plasticity in their responses to perforant-path stimulation (Buzsaki \& Eidelberg, 1982; Taube \& Schwartz- 
kroin, 1987), so levels of feed-forward inhibition in response to perforant-path stimulation could be changing during these sessions as well. Thus, it is not completely surprising that spike-amplitude changes show a pattern not obviously consistent with either EPSP or spike-latency changes. In general, the close correspondence between increases in EPSP values and decreases in spike latency make it seem likely that EPSP growth is the result of the strengthening of perforant-path synapses, rather than a net hyperpolarization of the granule cells.

There are several mechanisms that are known to increase synaptic strength, including PTP (a transient increase in the probability of transmitter release that results from high-frequency synaptic activation; see McNaughton, 1982) and LTE (see introduction). The design of the present experiments cannot distinguish between these or other possibilities.

Relationship of the present findings to others in the literature. Earlier reports from this laboratory have described another type of environmentally induced change in perforant path-evoked dentate granule-cell responses (Sharp et al., 1987, 1985). This change consisted of an increase in the population-spike component of the response, which developed gradually over several days of extensive free exploration in a spatially complex environment. When the enrichment treatment was discontinued, this increase decayed gradually over a period of weeks. Similar spike increases have been found to result after training in a brightness-discrimination task (Ruthrich et al., 1982), and in operant (Skelton et al., 1987) and classical conditioning tasks (Weisz, 1982). Unpublished observations by the present authors indicate that these spike changes and the EPSP changes reported here appear to operate largely independently of one another.

It is worth considering, in this context, the possible relationship between each of these two types of plasticity and the LTE induced by high-frequency electrical stimulation. The spike increase mentioned above is similar to LTE both in its overall decay-time course and in the fact that it decays more rapidly in old animals than in young animals, as does LTE (Barnes, 1979; Barnes \& McNaughton, 1985). It is unlike LTE, however, in that it has been seen to occur reliably only in the spike component, and not in the EPSP component of the response.

Conversely, the changes reported here were observed in the EPSP component of the response, as were those of LTE, but their decay-time course was much shorter than that often reported. However, the LTE time course can be on the order of minutes in some cases, and has been shown to be quite variable, depending on the exact conditions used to induce it (see, e.g., Barnes, 1979; Gustafsson, Wigström, Abraham, \& Haung, 1987). Thus, both the spike changes mentioned above and the EPSP changes reported here have some characteristics in common with LTE and may share overlapping mechanisms. It is possible that the high-frequency stimulation used to induce LTE actually results in several separable types of plasticity, as has been suggested by other workers (Andersen, Sundberg, Sveen, Swann, \& Wigström, 1980; Bliss \& Lomo, 1973; Douglas \& Goddard, 1975; Taube \& Schwartzkroin, 1988; Wilson, 1981; Wilson, Levy, \& Steward, 1981) and that under natural circumstances these may occur in isolation, and with very different time courses. Of course, it is also possible that other, as yet unknown, processes are responsible for these environmentally induced changes.

Several recent studies have shown that sensory-evoked responses in the dentate (e.g., West, Christian, Robinson, \& Deadwyler, 1982), as well as the firing patterns of hippocampal place cells (Sharp, Muller, \& Kubie, 1988; Wible et al., 1986), are, at least under some circumstances, dependent largely on the very recently past experience of the animal. It is possible that these results could be due to plasticity of the sort described here either in the dentate or in other parts of the hippocampal circuitry.

As in the present study, Winson and Abzug (1977, 1978) have reported changes in dentate-evoked responses as a function of animals' behavior. Differences between those studies and the present one in behavioral paradigm and methods of data analysis make comparison of the two difficult. However, the two data sets are not incompatible with each other. Winson and Abzug reported that EPSP values are similar during still-alertness and theta behavior, whereas we report that theta behavior has cumulative effects over time on this measure, and that these effects outlast the behavior itself. Since Winson and $A b$ zug did not report EPSP values over time during bouts of theta behavior or still-alertness, it is consistent with the present findings that, depending on when their measures were taken, similar values between the two states could be obtained. In addition, Winson and Abzug reported that spike values are higher during theta behavior than during still-alertness. This is consistent with the present observations, in that spike values were often higher immediately after an animal was transferred to a new environment, when theta behavior was also elevated. As mentioned above, this behavioral correlate may explain part of the pattern of spike changes seen here.

Possible role in learning and memory. The most important question with regard to the EPSP and spike alterations reported here is whether they reflect mnemonic processes in the perforant-path dentate-gyrus system. As mentioned above, data presented by Green et al. (in press) have suggested that the changes do not result simply from motor activity or theta activity per se. This leaves the possibility that they are strictly correlated with the environmental sensory input associated with exploratory behavior. If these changes are related to environmental input, it remains to be seen whether they reflect alterations in specific subsets of cellular elements having relationships to particular environmental inputs, or whether they result from 
a relatively uniform, nonspecific change across the population. Only in the former case could a significant amount of environmental information be encoded.

Despite this caveat, the changes reported here, along with the longer lasting, environmentally induced changes reported earlier (Sharp et al., 1987, 1985), suggest the possibility that the hippocampal formation may provide at least two independent memory stores, either or both of which may be used in a variety of behaviors in which the hippocampal formation plays a role.

\section{REFERENCES}

AbrahaM, W. C., Goddard, G. V. (1982). Modulation of synaptic transmission and LTP in the rat dentate gyrus by stimulation in and near the locus coeruleus. Society for Neuroscience Abstracts, 8, 482.

Alvarez-LeEFMANS, F. J., \& Gardner-Medwin, A. R. (1975). Influences of the septum on the hippocampal dentate area which are unaccompanied by field potentials. Journal of Physiology, 249, 14P-16P.

Andersen, P., Sundberg, S. H., Sveen, O., Swann, J. N., Wigström, H. (1980). Possible mechanisms for long-lasting potentiation of synaptic transmission in hippocampal slices in guinea-pigs. Journal of Physiology, 302, 463-482.

Assaf, S. Y., MAson, S. T., Mrllek, J. J. (1979). Noradrenergic modulation of neuronal transmission between the entorhinal cortex and the dentate gyrus of the rat. Journal of Physiology, 292, 52P.

AssAF, S. Y., Miller, J. J. (1978). Neuronal transmission in the dentate gyrus: Role of inhibitory mechanisms. Brain Research, 151, 587-592.

Barnes, C. A. (1979). Memory deficits associated with senescence: A neurophysiological and behavioral study in the rat. Joumal of Comparative \& Physiological Psychology, 93, 74-104.

Barnes, C. A., McNaughton, B. L. (1980). Spatial memory and hippocampal synaptic plasticity in senescent and middle-aged rats. In D. O. Stein (Ed.), Psychobiology of aging: Problems and perspectives (pp. 253-272). Amsterdam: Elsevier.

BARNES, C. A., MCNAughton, B. L. (1985). An age comparison of the rates of acquisition and forgetting of spatial information in relation to long-term enhancement of hippocampal synapses. Behavioral Neuroscience, 99, 1040-1048.

Buss, T. V. P., GARDNER-MedWIN, A. R. (1973). Long-lasting potentiation of synaptic transmission in the dentate area of the unanaesthetized rabbit following stimulation of the perforant path. Journal of Physiology, 232, 357-374.

Buss, T. V. P., Lomo, T. (1973). Long-lasting potentiation of synaptic transmission in the dentate area of the anaesthetized rabbit following stimulation of the perforant path. Journal of Physiology, 232, 331-356.

Buzsaki, G., Eidelberg, E. (1982). Direct afferent excitation and long-term potentiation of hippocampal interneurons. Journal of Neurophysiology, 48, 597-607.

DAHL, D., \& Winson, J. (1986). Influence of neurons of the parafascicular region on neuronal transmission from perforant pathway through dentate gyrus. Brain Research, 377, 391-396.

Douglas, R. M., GodDaRD, G. V. (1975). Long-term potentiation of the perforant path-granule cell synapse in the rat hippocampus. Brain Research, 86, 205-215.

Fantie, B. D., Goddard, G. V. (1982). Septal modulation of the population spike in the fascia dentata produced by perforant path stimulation in the rat. Brain Research, 252, 227-237.

Green, E. J., Barnes, C. A., McNaughton, B. L. (1986). Behavioral modulation of septal influences on hippocampal excitability. Society for Neuroscience Abstracts, 12, 1529.

Green, E. J., McNaughton, B. L., Barnes, C. A. (in press). Exploration-dependent modulation of evoked responses in fascia dentata: Dissociation of motor EEG and sensory factors, and evidence for a synaptic conductance change. Journal of Neuroscience.
Gustafsson, B., Wigström, H., Abraham, W. C., \& Haung, Y.-Y. (1987). Long-term potentiation in the hippocampus using depolarizing current pulses as the conditioning stimulus to single volley synaptic potentials. Journal of Neuroscience, 7, 774-780.

JARRARD, L. E. (1983). Selective hippocampal lesions and behavior: Effects of kainic acid lesions on performance of place and cue tasks. Behavioral Neuroscience, 97, 873-889.

MArR, D. (1971). Simple memory: A theory for archicortex. Proceedings of the Royal Society of London, B262, 23-81.

MCNaughton, B. L. (1982). Long-term synaptic enhancement and short-term potentiation in rat fascia dentata act through different mechanisms. Joumal of Physiology, 324, 249-262.

MCNaughton, B. L. (1983). Activity dependent modulation of hippocampal synaptic efficacy: Some implications for memory processes. In W. Seifert (Ed.), The neurobiology of the hippocampus (pp. 233251). London: Academic Press.

McNaughton, B. L., Barnes, C. A., Rao, G., Baldwin, J., * RASMUSSEN, M. (1986). Long-term enhancement of hippocampal synaptic transmission and the acquisition of spatial information. Jourmal of Neuroscience, 6, 563-571.

MCNaughton, B. L., Douglas, R. M., Goddard, G. V. (1978). Synaptic enhancement in the fascia dentata: Cooperativity among coactive afferents. Brain Research, 157, 277-293.

McNaughton, B. L., \& Morris, R. G. M. (1987). Hippocampal synaptic enhancement and information storage within a distributed memory system. Trends in Neuroscience, 10, 408-415.

Morris, R. G. M., ANDerson, E., LYNCH, G., BAudry, M. (1986). Selective impairment of long-term potentiation by an N-methyl-Daspartate receptor antagonist, AP-5. Nature, 319, 774-776.

Morris, R. G. M., Garrud, P., Rawlins, J. N. P., O'Keefe, J. (1982). Place navigation impaired in rats with hippocampal lesions. Nature, 297, 681-683.

Morris, R. G. M., Hagan, J. J., Rawlins, J. N. P. (1986). Allocentric spatial learning by hippocampectomized rats: A further test of the "spatial mapping" and "working memory" theories of hippocampal function. Quarterly Joumal of Experimental Psychology, 38B, 365-395.

OKKefFe, J. (1976). Place units in the hippocampus of the freely-moving rat. Experimental Neurology, 51, 78-109.

O'KeEFE, J., \& Dostrovsky, J. (1971). The hippocampus as a spatial map: Preliminary evidence from unit activity in the freely moving rat. Brain Research, 34, 171-175.

OKEEFE, J., \& NADEL, L. (1978). The hippocampus as a cognitive map. New York: Oxford University Press.

Olton, D. S., Becker, J. T., Handlemann, G. E. (1980). Hippocampal function: Working memory or cognitive mapping? Physiological Psychology, 8, 239-246.

Olton, D. S., WALKeR, J. W., \& GAGE, F. H. (1978). Hippocampal connections and spatial discrimination. Brain Research, 139, 295-308.

RAwLins, J. N. P. (1985). Associations across time: The hippocampus as a temporary memory store. Behavioral Brain Science, 8, 479-496.

Robinson, G. B., \& RACiNe, R. J. (1986). Interactions between septal and entorhinal inputs to the dentate gyrus: Facilitation effects. Brain Research, 379, 63-67.

Ruthrich, H., MatThIEs, H., \& OTT, T. (1982). Long-term changes in synaptic excitability of hippocampal cell populations as a result of training. In C. A. Marsan \& H. Matthies (Eds.), Neuronal plasticity and memory formation (pp. 589-594). New York: Raven Press.

Sharp, P. E., Barnes, C. A., \& McNaughton, B. L. (1987). Effects of aging on environmental modulation of hippocampal evoked responses. Behavioral Neuroscience, 101, 170-178.

Sharp, P. E., McNaughton, B. L., Barnes, C. A. (1985). Enhancement of hippocampal field potentials in rats exposed to a novel, complex environment. Brain Research, 339, 361-365.

Sharp, P. E., Muller, R. U., Kubie, J. L. (1988). Hippocampal place cells can fire differently in two visually identical locations in a symmetrical chamber. Society for Neuroscience Abstracts, 14, 126. Skelton, R. W., Scarth, A. S., Wilkie, D. M., Miller, J. J., \& PHILIPS, A. G. (1987). Long-term increases in dentate granule cell responsivity accompany operant conditioning. Joumal of Neuroscience, 7, 3081-3087. 
Sutherland, R. J., Wishaw, I. Q., KolB, B. (1983). A behavioral analysis of spatial localization following electrolytic, kainate- or colchicine-induced damage. Behavioral Brain Research, 7, 133-153.

Swanson, L. W., Teyler, T. J., Thompson, R. F. (1982). Hippocampal long-term potentiation: Mechanisms and implications for memory. Neurosciences Research Program Bulletin, 20, 613-765.

TAUBE, J. S., SChWARTZKRoIN, P. A. (1987). Intracellular recording from hippocampal CAl intemeurons before and after development of long-term potentiation. Brain Research, 419, 32-38.

Taube, J. S., \& SChwarTzKroIN, P. A. (1988). Mechanisms of longterm potentiation: EPSP/spike dissociation, intradendritic recordings, and glutamate sensitivity. Journal of Neuroscience, 8, 1632-1644.

VANDERWOLF, C. H. (1969). Hippocampal electrical activity and voluntary movement in the rat. Electroencephalography \& Clinical Neurophysiology, 26, 407-418.

WeISz, D. J. (1982). Activity of the dentate gyrus during nictitating membrane conditioning in the rabbit. In C. D. Woody (Ed.), Conditioning: Representations of involved neural functions, advances in behavioral biology (pp. 131-146). New York: Plenum.

West, M. O., Christian, E., Robinson, J. H., \& Deadwyler, S. A. (1982). Evoked potentials in the dentate gyrus reflect the retention of past sensory events. Neuroscience Letters, 28, 319-324.

Wible, C. G., Findung, R. L., Shapiro, M., Lang, E. J., Crane, S., Olton, D. S. (1986). Mnemonic correlates of unit activity in the hippocampus. Brain Research, 399, 97-110.
WILSON, R. C. (1981). Changes in translation of synaptic excitation to dentate granule cell discharge accompanying long-term potentiation. 1. Differences between normal and reinnervated dentate gyrus. Journal of Neurophysiology, 46, 324-338.

Wilson, R. C., LevY, W. B., STewarD, O. (1981). Changes in translation of synaptic excitation to granule cell discharge accompanying long-term potentiation. 2. An evaluation of mechanisms utilizing dentate gyrus dually innervated by surviving ipsilateral and sprouted crossed tempero-dentate inputs. Journal of Neurophysiology, 46, 339-355.

WINSON, J. (1980). Influence of raphe nuclei on neuronal transmission from perforant path through dentate gyrus. Joumal of Neurophysiology, 44, 937-950.

WINSON, J., ABZUG, C. (1977). Gating of neuronal transmission in the hippocampus: Efficacy of transmission varies with behavioral state. Science, 196, 1223-1225.

Winson, J., \& ABZug, C. (1978). Neuronal transmission through hippocampal pathways dependent on behavior. Joumal of Neurophysiology, 41, 716-732.

Winson, J., \& DAHL, D. (1985). Action of norepinephrine in the dentate gyrus. 1. Stimulation of locus coenuleus. Experimental Brain Research, 59, 491-496.

(Manuscript received November 29, 1988; revision accepted for publication April 27, 1989.) 